\title{
Optimal Ultrasonographic Measurements for Diagnosing Carpal Tunnel Syndrome in Patients With Diabetic Sensorimotor Polyneuropathy: A Case-Control Study
}

\author{
Ji Woong Park, MD, PhD, Seokmin Lee, MD, Rhee Wook Jang, MD, Sungche Lee, MD, \\ Sanghoon Lee, MD, Hyunchul Cho, MD, Yoon-Hee Choi, MD, PhD, Jinmyoung Kwak, MD
}

Department of Physical Medicine and Rehabilitation, Soonchunhyang University Seoul Hospital, Soonchunhyang University College of Medicine, Seoul, Korea

\begin{abstract}
Objective To investigate the optimal sonographic method for diagnosing carpal tunnel syndrome (CTS) in patients with diabetic sensorimotor polyneuropathy (DSP).

Methods A total of 190 participants were divided into four groups based on DSP history and electrodiagnostic results of CTS. The absolute parameters were measured at baseline and the relative values were calculated: maximal cross-sectional area (CSA) of the median nerve throughout the carpal tunnel (Mmax), median nerve CSA at the forearm level (Mf), ulnar nerve CSA at the pisiform level (Upi), difference between Mmax and $\mathrm{Mf}(\Delta \mathrm{MM})$, and difference between Mmax and Upi $(\Delta \mathrm{MU})$. Then, the optimal ultrasonographic parameters for diagnosing CTS, according to the presence of DSP, using absolute and relative cutoff values were analyzed.

Results Median and ulnar nerve CSAs were significantly larger in the DSP group than in the control group. In the DSP participants, the mean Mmax, $\triangle \mathrm{MM}$, and $\triangle \mathrm{MU}$ values were significantly larger in patients with both DSP and CTS than in patients with DSP only. The Mmax thresholds of $9.5 \mathrm{~mm}^{2}$ in the control group and $11.5 \mathrm{~mm}^{2}$ in the DSP group showed the greatest sensitivity and specificity for diagnosing CTS. The $\Delta$ MM thresholds of $2.5 \mathrm{~mm}^{2}$ and $\triangle \mathrm{MU}$ thresholds of $4.5 \mathrm{~mm}^{2}$ had the greatest sensitivity and specificity in both the DSP and control groups.

Conclusion Measurement of Mmax, $\triangle \mathrm{MM}$ and $\Delta \mathrm{MU}$ is an optimal ultrasonographic evaluation method for diagnosing CTS in patients with DSP.
\end{abstract}

Keywords Ultrasonography, Carpal tunnel syndrome, Diabetic neuropathies, Median nerve, Electromyography

Received July 27, 2018; Accepted October 5, 2018

Corresponding author: Jinmyoung Kwak

Department of Physical Medicine and Rehabilitation, Soonchunhyang University Seoul Hospital, 59 Daesagwan-ro, Yongsan-gu, Seoul 04401, Korea. Tel: +82-2-710-3148, Fax: +82-2-709-9265, E-mail: 115881@schmc.ac.kr

ORCID: Ji Woong Park (http://orcid.org/0000-0001-7987-3581); Seokmin Lee (http://orcid.org/0000-0003-3029-2174); Rhee Wook Jang (http://orcid. org/0000-0002-3741-8238); Sungche Lee (http://orcid.org/0000-0001-5324-7777); Sanghoon Lee (http://orcid.org/0000-0003-3007-7431); Hyunchul Cho (http://orcid.org/0000-0001-5103-0507); Yoon-Hee Choi (http://orcid.org/0000-0001-6651-7908); Jinmyoung Kwak (http://orcid.org/0000-00033606-755X).

(c) This is an open-access article distributed under the terms of the Creative Commons Attribution Non-Commercial License (http://creativecommons.org/ licenses/by-nc/4.0) which permits unrestricted noncommercial use, distribution, and reproduction in any medium, provided the original work is properly cited. Copyright $\odot 2019$ by Korean Academy of Rehabilitation Medicine 


\section{INTRODUCTION}

Carpal tunnel syndrome (CTS) is a common medical condition, in which the median nerve is compressed at the wrist, leading to paresthesia, numbness, and muscle weakness of the hand [1]. Diabetic sensorimotor polyneuropathy (DSP) is known to be a length-dependent, symmetrical polyneuropathy that mainly affects the large nerve fibers within the body $[2,3]$. The prevalence of CTS was reported to be $2 \%$ in the general population, $14 \%$ in diabetic patients without DSP, and $30 \%$ in diabetic patients with DSP [4]. CTS is traditionally diagnosed based on a combination of history of symptoms, physical examination, and electrodiagnostic study [1]. However, ultrasonography (US) has also played an adjuvant role in the diagnosis of CTS by evaluating the enlargement of the cross-sectional area (CSA) of the median nerve and the abnormality of the adjacent soft tissue $[5,6]$. Several studies have attempted to determine the absolute cutoff values of median nerve CSA for diagnosing CTS, but no consensus regarding the absolute diagnostic value has been established yet [5,7-9]. However, since absolute CSA can be affected by anthropometric character, sex, and presence of polyneuropathy, the relative CSA value is theoretically more accurate in the diagnosis of CTS. According to a report, the peripheral nerves of patients with DSP show diffuse enlargement at the whole nerve length, which represents sonographically larger CSA of the peripheral nerves [10]. Moreover, in a study of median nerve US in diabetic patients, the median nerve CSA in the carpal tunnel of DSP patients was greater than that in healthy individuals or in patients without DSP [11]. In patients with CTS combined with DSP, the focal enlargement process may also be added to the already enlarged median nerve. Thus, measuring only the absolute CSA of the median nerve may render false-positive results when diagnosing CTS combined with DSP.

Therefore, the objective of this study was to investigate the optimal US evaluation method for diagnosing CTS in patients with DSP.

\section{MATERIALS AND METHODS}

\section{Participants}

This prospective study was composed of two similar case-control designs and performed at Soonchunhyang
University Hospital, Seoul, Korea, from March 2010 to February 2011. The study was approved by the Institutional Review Board of the Soonchunhyang University Hospital (No. SCHUH 2009-010). Written informed consent was obtained from all participants before their participation in the study began. Age, sex, weight, height, and body mass index (BMI) were collected for all participants. This study initially enrolled 80 healthy controls, 80 patients with suspected CTS, and 80 patients with confirmed DSP. The participants in the DSP group were confirmed to have DSP by previous electrodiagnosis. Nerve conduction studies (NCS) were performed in both upper and lower limbs to diagnose DSP. Based on a previous guideline [12], the diagnostic criteria for DSP were defined as a sural sensory action potential of $<7.3 \mu \mathrm{V}$, and a peroneal motor conduction velocity of $<43.9 \mathrm{~m} / \mathrm{s}$, observed at the same time during the NCS. The suspected CTS participants were selected according to a history of CTS and clinical symptoms, including numbness and tingling sensation in the lateral four fingers, with or without thumb weakness. Participants with confirmed DSP and suspected CTS underwent physical examination and electrodiagnostic studies to confirm their CTS status and exclude other disease conditions that might influence peripheral nervous function. The participants who had a history of wrist surgery, fracture, and neurologic disorders (e.g., other peripheral polyneuropathies, ulnar neuropathy, radiculopathy, myelopathy, stroke), which may cause numbness or paresthesia in the limbs, were excluded. Among the 80 patients with suspected CTS, 42 had normal findings, 5 were diagnosed with cervical radiculopathy, and 33 showed findings consistent with CTS. Among the 80 patients with DSP, 2 were diagnosed with cervical radiculopathy, 1 was diagnosed with bilateral ulnar neuropathy, and 43 showed findings consistent with both CTS and DSP. A total of 190 patients were classified into four groups based on the electrophysiologic findings: group 1, patients with CTS and DSP; group 2, patients with DSP only; group 3, patients with CTS only; and group 4, healthy controls (Fig. 1). All patients underwent US examination after electrophysiologic evaluation during a visit.

\section{Ultrasonography}

US was performed using a 13.3-MHz linear array transducer, ProSound Alpha 7 (Hitachi-Aloka Medical, Tokyo, Japan). All measurements were taken by a single physi- 


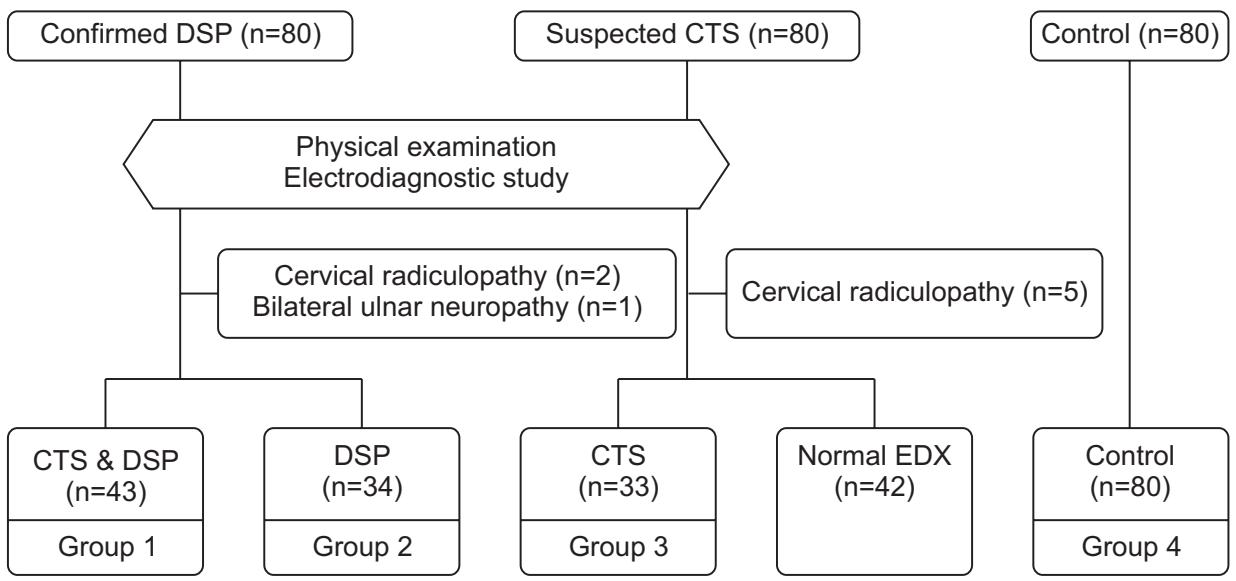

Fig. 1. Flow chart of the study. DSP, diabetic sensorimotor polyneuropathy; CTS, carpal tunnel syndrome; EDX, electrodiagnosis.

cian who had more than 5 years of US experience and was blinded to the diagnosis, physical findings, and electrodiagnostic testing results. The participants were comfortably placed in a supine position with arms extended, wrists resting on a flat surface, forearms in a supine position, and fingers in semi-extended position. Only the weight of the probe was applied to avoid causing any artificial nerve deformity. The angle of the ultrasound beam was kept perpendicular to the surface of the nerves and tendons to ensure the highest echogenic view. The CSAs of the median and ulnar nerves were measured at the inner border of the epineurial echogenic rim using a continuous tracing technique.

We scanned the CSA of the median (M) and ulnar (U) nerves in the axial plane at the following regions (Fig. 2): Mmax, maximally dilated median nerve CSA in the carpal tunnel from the hamate bone level to the radioulnar joint level through the pisiform bone level; Mf, median nerve CSA in the forearm at $10 \mathrm{~cm}$ proximal to the wrist crease; Upi, ulnar nerve CSA in the carpal tunnel at the pisiform bone level. The absolute CSA parameters were calculated in two relative values: $\triangle \mathrm{MM}$ (difference between Mmax and Mf) and $\Delta \mathrm{MU}$ (difference between Mmax and Upi).

\section{Electrodiagnostic evaluation}

The electrodiagnostic study was performed by two experienced physiatrists who were blinded to the patient's history and symptoms, using Medelec Synergy Mobile 5-channel (Oxford Instruments, Surrey, UK) devices. The evaluation process was performed under standard room temperature of $25^{\circ} \mathrm{C}$ and hand temperature of more than $32^{\circ} \mathrm{C}$. The motor NCS of the median nerve was recorded with surface electrodes from the abductor pollicis brevis

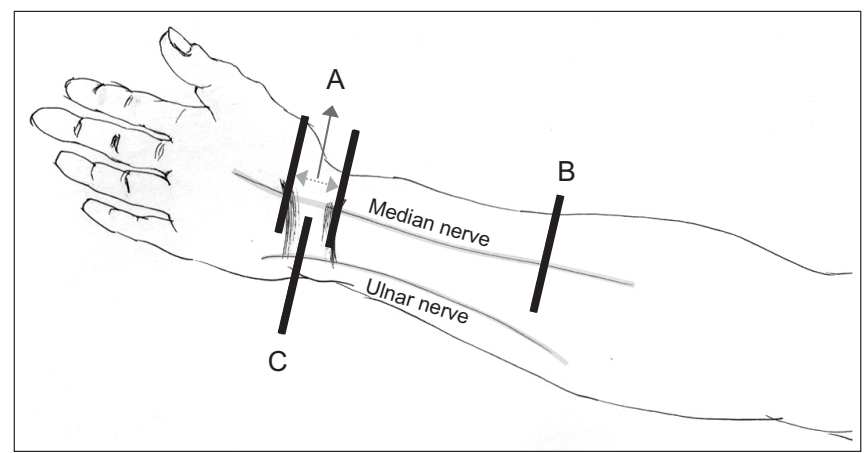

Fig. 2. The sonographically measured regions. A is maximally dilated median nerve cross-sectional area (CSA) in the carpal tunnel. B is median nerve CSA in the forearm at $10 \mathrm{~cm}$ proximal to the wrist crease. $C$ is ulnar nerve CSA in the carpal tunnel at the pisiform bone level.

muscle. Median motor nerve proximal and distal latencies, median motor nerve conduction velocity, and compound muscle action potential amplitudes at the wrist and elbow were measured. Median sensory NCSs were recorded with ring electrodes placed on the 3rd digit antidromically at 7 and $14 \mathrm{~cm}$ proximal to the recording electrode. Lumbrical-interosseous median-ulnar distal latency difference (LIMULD) was also measured to improve the diagnostic accuracy of CTS in patients with DSP. Median and ulnar motor NCSs were recorded with cup electrodes from the 2nd lumbrical-interosseous muscle. The stimulation points were proximal to the carpal tunnel and Guyon canal for the median and ulnar nerves, respectively, with a standard distance of $9 \mathrm{~cm}$. LIMULD was calculated by subtracting the ulnar motor distal latency from the median motor distal latency.

CTS was diagnosed if the results of the NCS fulfilled two of the following criteria [13,14]: (1) median motor 
nerve distal latency greater than 3.7 ms or median sensory nerve conduction velocity slower than $50 \mathrm{~m} / \mathrm{s}$; (2) an increase in the palm sensory nerve action potential amplitude of $>50 \%$ compared with wrist stimulation; (3) LIMULD more than $0.4 \mathrm{~ms}$.

The electrophysiologic severities of CTS were assessed using the NCS results, according to neurophysiologic classification [15]. Hands with CTS were divided into four groups based on neurophysiologic findings: 'mild, abnormal digit/wrist sensory nerve conduction velocity and normal distal motor latency; 'moderate', abnormal digit/wrist sensory nerve conduction velocity and abnormal distal/motor latency; 'severe', absence of sensory response and abnormal distal motor latency; and 'extreme', absence of motor and sensory responses.

\section{Statistical analysis}

To estimate the sample size, we referred to a previous study that determined the criteria for diagnosing the comorbidity of CTS with DSP using US parameters [16]. We used median nerve CSA to estimate the number of subjects needed. To investigate the optimal sonographic method with a power of $80 \%$ and two-sided test of $5 \%$, 52 patients had to be included in each group. With an estimated a drop-out rate of $30 \%$, a sample size of 80 in each group was calculated. All p-values less than 0.05 were considered to be statistically significant. Analysis of variance (ANOVA) and Student t-test were used to compare continuous variables, and the $\chi^{2}$ test was utilized to compare the categorical variables, such as sex and CTS grades. Receiver operating characteristic (ROC) curves were used to detect optimal US cutoff values for diagnosing CTS, with or without DSP. A pairwise comparison test was used to compare the differences between the ROC curves. All data were analyzed using SPSS version 20 (IBM, Armonk, NY, USA) and MedCalc version 13 (MedCalc Software, Mariakerke, Belgium).

\section{RESULTS}

The participants' characteristics are summarized in Table 1. There were no significant differences in terms of age, sex, weight, height, and BMI among the four groups. There were no significant differences in the CTS grade between groups 1 and 3, and in diabetes duration and $\mathrm{HbAlc}$ between groups 1 and 2 .

To investigate the influence of CTS and DSP on the CSA of the peripheral nerves, we compared the mean sonographic parameters between the CTS and non-CTS patients in each of the DSP and non-DSP groups (Fig. 3).

In the DSP group, group $1(n=43)$ showed larger primary US parameters than group $2(n=34)$ (Fig. $3 A)$ :

Table 1. Baseline demographics of participants

\begin{tabular}{|c|c|c|c|c|c|}
\hline & Group 1 & Group 2 & Group 3 & Group 4 & p-value \\
\hline Number of hands & 43 & 34 & 33 & 80 & \\
\hline Age (yr) & $63.3 \pm 9.4$ & $61.0 \pm 10.1$ & $59.9 \pm 6.3$ & $61.2 \pm 5.9$ & $0.29^{a)}$ \\
\hline Sex, female & $24(55.8)$ & $15(44.1)$ & $19(57.6)$ & $50(62.5)$ & $0.44^{\mathrm{b})}$ \\
\hline Height $(\mathrm{cm})$ & $161.1 \pm 6.4$ & $164.3 \pm 10.1$ & $160.3 \pm 6.9$ & $160.6 \pm 6.2$ & $0.07^{\mathrm{a})}$ \\
\hline Weight (kg) & $59.0 \pm 8.7$ & $62.0 \pm 10.1$ & $60.0 \pm 9.5$ & $59.2 \pm 8.6$ & $0.43^{\text {a) }}$ \\
\hline BMI $\left(\mathrm{kg} / \mathrm{m}^{2}\right)$ & $22.7 \pm 3.0$ & $23.3 \pm 5.0$ & $23.3 \pm 2.6$ & $22.9 \pm 2.6$ & $0.82^{\text {a) }}$ \\
\hline Diabetes duration (yr) & $17.2 \pm 8.7$ & $16.2 \pm 8.4$ & NA & NA & $0.61^{\mathrm{c})}$ \\
\hline $\operatorname{HbAlc}(\%)$ & $6.7 \pm 1.2$ & $6.3 \pm 0.9$ & NA & NA & $0.12^{\mathrm{c})}$ \\
\hline CTS grades & & & & & $0.11^{\mathrm{b})}$ \\
\hline Mild to moderate & $16(37.2)$ & NA & $18(54.5)$ & NA & \\
\hline Severe to extreme & $27(62.8)$ & NA & $15(45.5)$ & NA & \\
\hline
\end{tabular}

Values are presented as mean \pm standard deviation or number (\%).

BMI, body mass index; CTS, carpal tunnel syndrome; DSP, diabetic sensorimotor polyneuropathy; NA, not applicable; Group 1, patients with CTS and DSP; Group 2, patients with DSP; Group 3, patients with CTS only; Group 4, healthy controls.

${ }^{\text {a) }}$ One-way analysis of variance test.

${ }^{\text {b) }} \chi^{2}$ test for qualitative data.

${ }^{c}$ Student $t$-test for quantitative data. 
Mmax (13.8 vs. $9.0 \mathrm{~mm}^{2}$; $95 \%$ confidence interval [CI], 3.4-6.1; $\mathrm{p}<0.001$ ), $\triangle \mathrm{MM}$ (5.5 vs. $1.5 \mathrm{~mm}^{2}$; $95 \% \mathrm{CI}, 2.8-5.2$; $\mathrm{p}<0.001)$, and $\Delta \mathrm{MU}\left(7.4\right.$ vs. $3.1 \mathrm{~mm}^{2} ; 95 \% \mathrm{CI}, 2.9-5.7$; $\mathrm{p}<0.001$ ). However, the reference parameters ( $\mathrm{Mf}$ and Upi) were not different between groups 1 and 2: Mf (8.0 vs. $7.5 \mathrm{~mm}^{2}$; $95 \% \mathrm{CI}, 0.4-0.3 ; \mathrm{p}=0.204$ ), and Upi (6.4 vs. 5.9 $\mathrm{mm}^{2}$; 95\% CI, $-0.1-1.0 ; \mathrm{p}=0.120$ ).

In the non-DSP group, similar patterns were observed compared with the DSP group. Group $3(n=33)$ showed larger US variables than group $4(\mathrm{n}=80)$ (Fig. 3B): Mmax (12.9 vs. $8.0 \mathrm{~mm}^{2}$; $95 \% \mathrm{CI}, 3.6$ to $\left.6.2 ; \mathrm{p}<0.001\right), \Delta \mathrm{MM}(6.7$ vs. $1.6 \mathrm{~mm}^{2}$; $95 \% \mathrm{CI}, 3.7$ to $6.4 ; \mathrm{p}<0.001$ ), $\Delta \mathrm{MU}$ (7.8 vs. 2.9 $\mathrm{mm}^{2}$; $95 \% \mathrm{CI}, 3.6$ to $\left.6.1 ; \mathrm{p}<0.001\right)$. However, the reference parameters ( $\mathrm{Mf}$ and Upi) were not different between groups 3 and 4: $\mathrm{Mf}\left(6.2\right.$ vs. $6.3 \mathrm{~mm}^{2}$; 95\% CI, -0.6-0.4; $\mathrm{p}=0.705)$, and Upi (5.1 vs. $5.0 \mathrm{~mm}^{2} ; 95 \% \mathrm{CI},-0.3-0.5$; $\mathrm{p}=0.645$ ).

To investigate the influence of DSP on peripheral nerves, we compared the mean sonographic parameters between the DSP and non-DSP patients in each of the CTS and the non-CTS groups. Group $1(n=43)$ showed significantly larger reference parameters (Mf and Upi) compared with group 3 ( $\mathrm{n}=33$ ): $\mathrm{Mf}\left(8.0\right.$ vs. $6.2 \mathrm{~mm}^{2}$; $95 \%$
CI, 1.3-2.7; $\mathrm{p}=0.000$ ), and Upi ( 6.4 vs. $5.1 \mathrm{~mm}^{2}$; $95 \% \mathrm{CI}$, 0.7-1.8; $\mathrm{p}=0.000$ ). However, $\mathrm{Mmax}, \Delta \mathrm{MM}$, and $\Delta \mathrm{MU}$ did not show significant differences between groups 1 and 3: $\operatorname{Mmax}$ (13.8 vs. $12.9 \mathrm{~mm}^{2}$; $95 \% \mathrm{CI},-0.9-2.7 ; \mathrm{p}=0.330$ ), $\Delta \mathrm{MM}$ (5.5 vs. $6.7 \mathrm{~mm}^{2} ; 95 \% \mathrm{CI},-2.8-0.5 ; \mathrm{p}=0.181$ ), and $\Delta \mathrm{MU}$ (7.4 vs. $7.8 \mathrm{~mm}^{2}$; 95\% CI, 3.6-6.1; $\mathrm{p}=0.685$ ).

In the non-CTS group, group $2(\mathrm{n}=34)$ showed significantly larger US parameters than group $4(\mathrm{n}=80)$ : Mmax ( 9.0 vs. $8.0 \mathrm{~mm}^{2}$; $95 \% \mathrm{CI}, 0.5-1.5 ; \mathrm{p}=0.000$ ), $\mathrm{Mf}$ (7.5 vs. 6.3 $\mathrm{mm}^{2}$; 95\% CI, 0.6-1.7; $\mathrm{p}=0.000$ ), and Upi (6.4 vs. $5.1 \mathrm{~mm}^{2}$; 95\% CI, 0.5-1.3; $\mathrm{p}=0.000$ ). However, $\Delta \mathrm{MM}$ and $\Delta \mathrm{MU}$ did not show significant differences between groups 2 and 4: $\Delta \mathrm{MM}$ (1.5 vs. $1.6 \mathrm{~mm}^{2} ; 95 \% \mathrm{CI},-0.7-0.5 ; \mathrm{p}=0.671$ ) and $\Delta \mathrm{MU}$ (3.1 vs. $2.9 \mathrm{~mm}^{2}$; 95\% CI, $-0.5-0.7 ; \mathrm{p}=0.652$ ).

The ROC curve analysis was performed to identify the sensitivity and specificity of the US parameters in diagnosing CTS in the DSP and non-DSP groups (Fig. 4, Table 2). Based on the ROC curves analyses, the following Mmax, $\triangle \mathrm{MM}$ and $\Delta \mathrm{MU}$ cutoff values were chosen to diagnose CTS: Mmax thresholds that showed the greatest sensitivity and specificity were 9.5 and $11.5 \mathrm{~mm}^{2}$ in the non-DSP and DSP groups, respectively; $\triangle \mathrm{MM}$ and $\Delta \mathrm{MU}$ thresholds of 2.5 and $4.5 \mathrm{~mm}^{2}$, respectively, had the great-
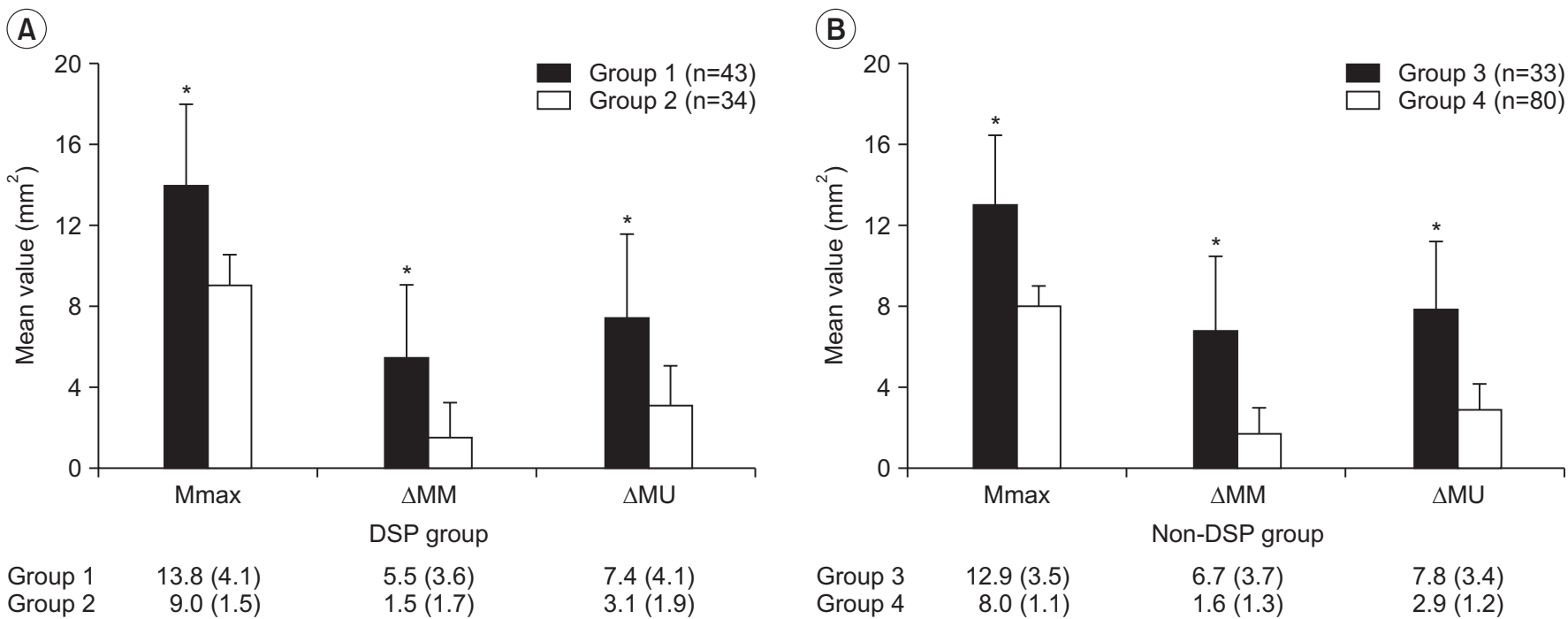

Fig. 3. The mean sonographic values were compared between patients with CTS and those without CTS, according to the presence of DSP: (A) DSP group and (B) non-DSP group. Data are expressed as mean \pm standard deviation. CTS, carpal tunnel syndrome; DSP, diabetic sensorimotor polyneuropathy; Mmax, maximal cross-sectional area of the median nerve throughout the carpal tunnel; $\Delta \mathrm{MM}$, difference between maximal cross-sectional area of the median nerve throughout the carpal tunnel and the cross-sectional area of the median nerve at the forearm level; $\Delta \mathrm{MU}$, difference between maximal cross-sectional area of the median nerve throughout the carpal tunnel and the cross-sectional area of the ulnar nerve at the pisiform level. * $\mathrm{p}<0.001$, using Student $\mathrm{t}$-test to compare the 2 means (group 1, patients with CTS and DSP; group 2, patients with DSP; group 3, patients with CTS only; group 4, healthy controls). 
est sensitivity and specificity in both the DSP and nonDSP groups. The diagnostic accuracy of each US evaluation method (Mmax, $\Delta \mathrm{MM}$, and $\Delta \mathrm{MU}$ ) was analyzed using pairwise comparison tests. In the DSP group, the areas under the ROC curves were 0.89 (95\% CI, 0.82-0.96) for Mmax, 0.87 (95\% CI, 0.79-0.95) for $\triangle \mathrm{MM}$, and 0.83
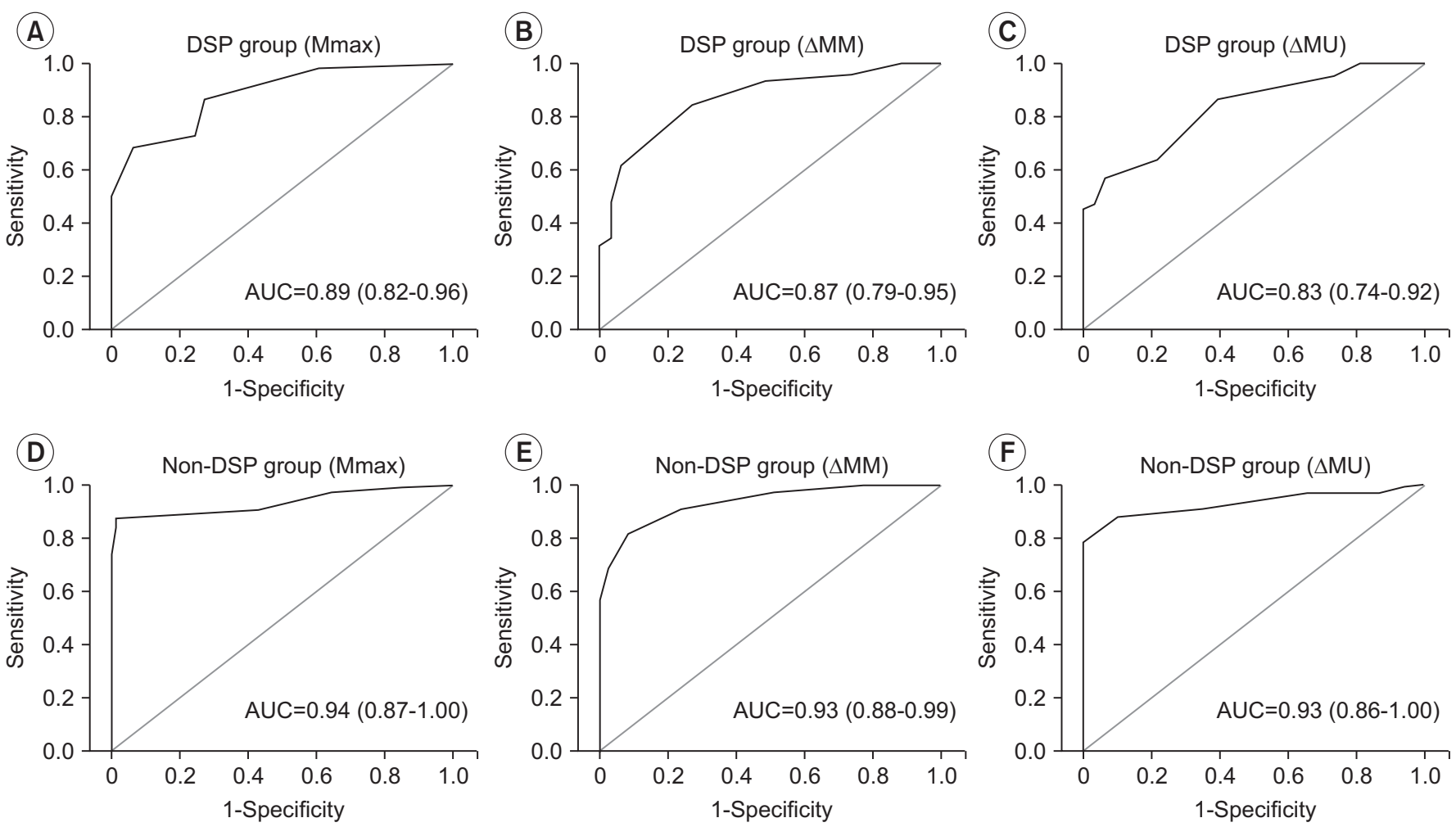

Fig. 4. The ROC curves of the ultrasound parameters (Mmax, $\triangle M M$, and $\triangle M U$ ) were measured in the DSP group and non-DSP group: (A) ROC curve of Mmax in the DSP group, (B) ROC curve of $\triangle M M$ in the DSP group, (C) ROC curve of $\triangle \mathrm{MU}$ in the DSP group, (D) ROC curve of Mmax in the non-DSP group, (E) ROC curve of $\triangle \mathrm{MM}$ in the non-DSP group, and (F) ROC curve of $\triangle \mathrm{MU}$ in the non-DSP group. ROC, receiver operating characteristic; DSP, diabetic sensorimotor polyneuropathy; Mmax, maximal cross-sectional area of the median nerve throughout the carpal tunnel; $\Delta \mathrm{MM}$, difference between maximal cross-sectional area of the median nerve throughout the carpal tunnel and the cross-sectional area of the median nerve at the forearm level; $\Delta \mathrm{MU}$, difference between maximal cross-sectional area of the median nerve throughout the carpal tunnel and the cross-sectional area of the ulnar nerve at the pisiform level; AUC, area under the curve.

Table 2. Sensitivity and specificity of the measuring parameters in diagnosing carpal tunnel syndrome

\begin{tabular}{|c|c|c|c|c|c|}
\hline & \multirow{2}{*}{$\begin{array}{c}\text { Cutoff value } \\
\left(\mathrm{mm}^{2}\right)\end{array}$} & \multicolumn{2}{|c|}{ DSP group $(n=77)$} & \multicolumn{2}{|c|}{ Non-DSP group $(n=113)$} \\
\hline & & Sensitivity (\%) & Specificity (\%) & Sensitivity (\%) & Specificity (\%) \\
\hline \multirow[t]{2}{*}{ Mmax } & 9.5 & 86 & 73 & 88 & 99 \\
\hline & 11.5 & 78 & 94 & 59 & 100 \\
\hline$\Delta \mathrm{MM}$ & 2.5 & 84 & 73 & 91 & 77 \\
\hline$\Delta \mathrm{MU}$ & 4.5 & 64 & 79 & 88 & 90 \\
\hline
\end{tabular}

DSP, diabetic sensorimotor polyneuropathy; Mmax, maximal cross-sectional area of the median nerve throughout the carpal tunnel; $\Delta \mathrm{MM}$, difference between maximal cross-sectional area of the median nerve throughout the carpal tunnel and cross-sectional area of the median nerve at the forearm level; $\Delta \mathrm{MU}$, difference between maximal crosssectional area of the median nerve throughout the carpal tunnel and cross-sectional area of the ulnar nerve at the pisiform level. 
(95\% CI, 0.74-0.92) for $\Delta \mathrm{MU}$. In the non-DSP group, the areas under the ROC curves were 0.94 (95\% CI, 0.87-1.00) for Mmax, 0.93 (95\% CI, 0.88-0.99) for $\triangle \mathrm{MM}$, and 0.93 (95\% CI, 0.86-1.00) for $\Delta \mathrm{MU}$. There was no statistically significant difference between the three methods in the DSP and non-DSP groups.

\section{DISCUSSION}

In our study, we determined that Mmax, $\triangle \mathrm{MM}$, and $\triangle \mathrm{MU}$ could be useful for diagnosing CTS in patients with DSP.

The mechanism of nerve swelling in diabetic neuropathy has been studied. The wet weights and water content of the sciatic nerves of diabetic rats were increased compared with those of normal rats [17]. It has been hypothesized that this increased water content is related to increased aldose reductase conversion of glucose to sorbitol [18]. In the peripheral nerve, aldose reductase has been localized to the Schwann cell [19]. The slow penetration of sorbitol of cell membranes [20] can result in osmotic swelling. Given this nerve enlargement in patients with diabetic neuropathy, US has been increasingly used for diagnosing diabetic neuropathy. In a previous study, the CSA of the median nerve of diabetic patients with DSP was significantly larger than that of normal healthy subjects [11]. In the present study, the Mmax of the median nerve had a tendency to be larger in the DSP group than in the non-DSP group.

Some studies have attempted to ascertain the usefulness of US for diagnosing CTS in patients with DSP. US can be performed non-invasively and easily compared with electrodiagnostic studies. In addition, treatment such as injection therapy can be conducted simultaneously with diagnosis, and adjacent structures can be evaluated if needed. A previous cross-sectional casecontrol study reported that the CSA of the median nerve of patients with CTS was similar to that of patients with CTS and DSP [21]. Another research study showed a similar result at the wrist level, but reported a significant difference in the CSA of the median nerve in the forearm level between patients with CTS only and those with CTS and DSP [16]. This result was considered to be due to the diffuse nerve enlargement from the wrist to the forearm in patients with DSP. Similar to previous studies, there were no significant differences in the US values between patients with CTS and DSP (group 1) and patients with CTS only (group 3) in the present study.

Some US parameters have been suggested for the diagnosis of CTS in patients with DSP. Some studies have reported that the wrist-to-forearm ratio (WFR) of the nerve diameter or CSA determined by US could be useful for diagnosing the comorbidity of CTS with DSP $[16,22]$. However, one of the studies reported that the WFR has a low diagnostic accuracy and should be used with caution [22] In addition, the study evaluating US for the diagnosis of CTS in patients with DSP reported that US parameters, including CSA, WFR, and wrist-forearm difference, were not significantly different between patients with CTS plus DSP and patients with DSP only [23]. Hence, the aforementioned ultrasound parameters cannot totally replace electrodiagnostic studies in diagnosis of CTS.

In this study, a significant difference was found in Mmax, $\triangle \mathrm{MM}$, and $\triangle \mathrm{MU}$ between patients with CTS and DSP (group 1) and patients with DSP only (group 2), which is contrary to the results of a previous study using the wrist-forearm difference. This discrepant outcome was considered to be due to the use of the maximal value in measuring the dilated median CSA in the present study. If measured at the fixed site, the CSA of the median nerve might not reflect the true neurophysiologic status of the nerve. Additionally, the CSA of the ulnar nerve could be a good standard parameter for diagnosing CTS in patients with DSP, because CTS mainly concerns pathogenesis of the median nerve, due to its location in the carpal tunnel.

In a study reporting the usefulness of US in diagnosing CTS in patients with DSP [16], the cutoff value of the median nerve CSA at the wrist level was $11.6 \mathrm{~mm}^{2}$. Similarly, the cutoff value of Mmax was $11.5 \mathrm{~mm}^{2}$ in this study. However, the sensitivity and specificity were higher in this study. Moreover, we determined that the cutoff value was $9.5 \mathrm{~mm}^{2}$ in the non-DSP group. Therefore, to diagnose CTS, the median nerve CSA should be measured as a maximal value through the carpal tunnel from the hamate bone level to the radioulnar joint level, and should be used as a different value in DSP and non-DSP groups.

The cutoff values of $\Delta \mathrm{MM}$ and $\Delta \mathrm{MU}$ for diagnosing CTS were 2.5 and $4.5 \mathrm{~mm}^{2}$, respectively. In the ROC curve analysis, the Mmax, $\Delta \mathrm{MM}$, and $\Delta \mathrm{MU}$ were not significantly different between the DSP and non-DSP groups. Furthermore, $\triangle \mathrm{MM}$ and $\Delta \mathrm{MU}$ can be obtained through 
simple subtraction.

There were some limitations to this study. First, we evaluated the Mmax among the CSAs values of median nerve at three levels: the hamate bone level, the pisiform bone level, and the radioulnar joint level. Thus, the maximal value among these three might not be the maximal value of the median nerve. Second, the US study was only conducted once by a single physiatrist. Thus, no interrater and intra-rater reliability tests could be performed. Future studies should be performed by multiple physiatrists with reliability tests. Third, we used a neurophysiologic classification to assess the electrophysiologic severity of CTS without considering DSP. However, pathologic alteration of peripheral nerves in patients with DSP might affect the results of CTS severity and lead to bias. Finally, we recruited patients with DSP whose diagnosis was confirmed by NCS within a year, without additional electrodiagnostic assessment for DSP after recruitment for this study. Thus, the neurophysiologic status of the nerves could differ between the times of diagnosis and recruitment. Therefore, we could not analyze the correlation between electrophysiologic parameters and US features.

In conclusion, US could be considered as an alternative method for diagnosing CTS in patients with DSP. The US parameters of Mmax, $\Delta \mathrm{MM}$, and $\Delta \mathrm{MU}$ are expected to be used with ease in clinical practice.

\section{CONFLICT OF INTEREST}

No potential conflict of interest relevant to this article was reported.

\section{ACKNOWLEDGMENTS}

This work was supported by the Soonchunhyang University Research Fund.

\section{REFERENCES}

1. Gelberman RH, Eaton RG, Urbaniak JR. Peripheral nerve compression. Instr Course Lect 1994;43:31-53.

2. Dyck PJ, Kratz KM, Karnes JL, Litchy WJ, Klein R, Pach JM, et al. The prevalence by staged severity of various types of diabetic neuropathy, retinopathy, and nephropathy in a population-based cohort: the Roch- ester Diabetic Neuropathy Study. Neurology 1993;43: 817-24.

3. Dyck PJ, Albers JW, Andersen H, Arezzo JC, Biessels GJ, Bril V, et al. Diabetic polyneuropathies: update on research definition, diagnostic criteria and estimation of severity. Diabetes Metab Res Rev 2011;27:620-8.

4. Atroshi I, Gummesson C, Johnsson R, Ornstein E, Ranstam J, Rosen I. Prevalence of carpal tunnel syndrome in a general population. JAMA 1999;282:153-8.

5. Wong SM, Griffith JF, Hui AC, Lo SK, Fu M, Wong KS. Carpal tunnel syndrome: diagnostic usefulness of sonography. Radiology 2004;232:93-9.

6. Abrishamchi F, Zaki B, Basiri K, Ghasemi M, Mohaghegh M. A comparison of the ultrasonographic median nerve cross-sectional area at the wrist and the wrist-to-forearm ratio in carpal tunnel syndrome. J Res Med Sci 2014;19:1113-7.

7. Koyuncuoglu HR, Kutluhan S, Yesildag A, Oyar O, Guler K, Ozden A. The value of ultrasonographic measurement in carpal tunnel syndrome in patients with negative electrodiagnostic tests. Eur J Radiol 2005;56: 365-9.

8. Lee D, van Holsbeeck MT, Janevski PK, Ganos DL, Ditmars DM, Darian VB. Diagnosis of carpal tunnel syndrome: ultrasound versus electromyography. Radiol Clin North Am 1999;37:859-72.

9. Wiesler ER, Chloros GD, Cartwright MS, Smith BP, Rushing J, Walker FO. The use of diagnostic ultrasound in carpal tunnel syndrome. J Hand Surg Am 2006;31:726-32.

10. Yasuda H, Sanada M, Kitada K, Terashima T, Kim H, Sakaue Y, et al. Rationale and usefulness of newly devised abbreviated diagnostic criteria and staging for diabetic polyneuropathy. Diabetes Res Clin Pract 2007;77 Suppl 1:S178-83.

11. Watanabe T, Ito H, Morita A, Uno Y, Nishimura T, Kawase $H$, et al. Sonographic evaluation of the median nerve in diabetic patients: comparison with nerve conduction studies. J Ultrasound Med 2009;28:727-34.

12. Hsu WC, Chiu YH, Chen WH, Chiu HC, Liou HH, Chen TH. Simplified electrodiagnostic criteria of diabetic polyneuropathy in field study (KCIS No. 14). Neuroepidemiology 2007;28:50-5.

13. Yagci I, Gunduz OH, Sancak S, Agirman M, Mesci E, Akyuz G. Comparative electrophysiological techniques in the diagnosis of carpal tunnel syndrome in 
patients with diabetic polyneuropathy. Diabetes Res Clin Pract 2010;88:157-63.

14. Werner RA, Andary M. Electrodiagnostic evaluation of carpal tunnel syndrome. Muscle Nerve 2011;44:597607.

15. Giannini F, Cioni R, Mondelli M, Padua R, Gregori B, D'Amico P, et al. A new clinical scale of carpal tunnel syndrome: validation of the measurement and clinical-neurophysiological assessment. Clin Neurophysiol 2002;113:71-7.

16. Kim LN, Kwon HK, Moon HI, Pyun SB, Lee HJ. Sonography of the median nerve in carpal tunnel syndrome with diabetic neuropathy. Am J Phys Med Rehabil 2014;93:897-907.

17. Jakobsen J. Peripheral nerves in early experimental diabetes: expansion of the endoneurial space as a cause of increased water content. Diabetologia 1978;14:1139.

18. Lee D, Dauphinee DM. Morphological and functional changes in the diabetic peripheral nerve: using diagnostic ultrasound and neurosensory testing to select candidates for nerve decompression. J Am Podiatr
Med Assoc 2005;95:433-7.

19. Gabbay KH. Role of sorbitol pathway in neuropathy. In: Camerini-Dgvalos RA, Cole HS, editors. Vascular and neurological changes in early diabetes. New York: Academic Press; 1973. p. 417-24.

20. Lefevre PG, Davies RI. Active transport into the human erythrocyte; evidence from comparative kinetics and competition among monosaccharides. J Gen Physiol 1951;34:515-24.

21. Kotb MA, Bedewi MA, Aldossary NM, Mahmoud G, Naguib MF. Sonographic assessment of carpal tunnel syndrome in diabetic patients with and without polyneuropathy. Medicine (Baltimore) 2018;97:e11104.

22. Steinkohl F, Loizides A, Gruber L, Karpf M, Morsdorf G, Gruber I, et al. Ultrasonography for the diagnosis of carpal tunnel syndrome in diabetic patients: missing the mark? Rofo 2018.

23. Hassan A, Leep Hunderfund AN, Watson J, Boon AJ, Sorenson EJ. Median nerve ultrasound in diabetic peripheral neuropathy with and without carpal tunnel syndrome. Muscle Nerve 2013;47:437-9. 